\title{
First report of Theileria and Anaplasma in the Mongolian gazelle, Procapra gutturosa
}

\author{
Youquan $\mathrm{Li}^{1,2^{*}}$, Ze Chen ${ }^{1,2}$, Zhijie Liu ${ }^{1,2}$, Junlong Liu ${ }^{1,2}$, Jifei Yang ${ }^{1,2}$, Qian $\mathrm{Li}^{1}$, Yaqiong Li ${ }^{1}$, Qiaoyun Ren ${ }^{1,2}$, \\ Qingli Niu ${ }^{1,2}$, Guiquan Guan ${ }^{1,2}$, Jianxun Luo ${ }^{1,2}$ and Hong Yin ${ }^{1,2}$
}

\begin{abstract}
Background: Theileria and Anaplasma are especially important emerging tick-borne pathogens of animals and humans. Molecular surveys and identification of the infectious agents in Mongolian gazelle, Procapra gutturosa are not only crucial for the species preservation, but also provide valuable information on parasite and bacterial epidemiology.

Findings: A molecular surveillance study was undertaken to assess the prevalence of Theileria spp. and Anaplasma spp. in $P$. gutturosa by PCR in China. Theileria luwenshuni, A. bovis, A. phagocytophilum, and A. ovis were frequently found in P. gutturosa in China, at a prevalence of $97.8 \%, 78.3 \%, 65.2 \%$, and $52.2 \%$, respectively. The prevalence of each pathogens in the tick Haemaphysalis longicornis was $80.0 \%, 66.7 \%, 76.7 \%$, and $0 \%$, respectively, and in the tick Dermacentor niveus was $88.2 \%, 35.3 \%, 88.2 \%$, and 58.5\%, respectively. No other Theileria or Anaplasma species was found in these samples. Rickettsia raoultii was detected for the first time in P. gutturosa in China.
\end{abstract}

Conclusions: Our results extend our understanding of the epidemiology of theileriosis and anaplasmosis in P. gutturosa, and will facilitate the implementation of measures to control these tick-borne diseases in China.

Keywords: Theileria, Anaplasma, Detection, Procapra gutturosa, PCR, China

\section{Findings}

\section{Background}

Theileria is mainly transmitted by tick vectors and cause heavy economic losses to the livestock industry. The family Anaplasmataceae in the order Rickettsiales was reclassified in 2001, and includes several genera, including Anaplasma, Ehrlichia, Neorickettsia, and Wolbachia. Of them, the genera Anaplasma and Ehrlichia are especially important as emerging tick-borne pathogens in both humans and animals [1]. Anaplasma phagocytophilum is the causative agent of human granulocytic anaplasmosis, an extremely dangerous disease associated with high mortality rates in humans [2-4]. Other Anaplasma spp., such as A. bovis, A. ovis, A. marginale, and A. centrale, infect the erythrocytes and other cells of ruminants $[3,4]$. Anaplasmosis is endemic in tropical and subtropical areas, but

\footnotetext{
* Correspondence: youquan-li@163.com

'State Key Laboratory of Veterinary Etiological Biology, Key Laboratory of Veterinary Parasitology of Gansu Province, Lanzhou Veterinary Research Institute, Chinese Academy of Agricultural Sciences, Xujiaping 1, Lanzhou, Gansu 730046, PR China

${ }^{2}$ Jiangsu Co-innovation Center for Prevention and Control of Important Animal Infectious Diseases and Zoonoses, Yangzhou 225009, Peoples Republic of China
}

is also frequently reported in temperate regions. Six or seven Anaplasma species have been reported in North America, Europe, Africa, and Asia [5-11], and some have also been reported in sheep, goats, and cattle throughout China $[9,12,13]$.

The detection and isolation of Theileria and Anaplasma require specialized laboratories staffed by technicians with a high degree of expertise, primarily because the species life cycles are intracellular. Several sensitive molecular tools, such as PCR, have been used to detect and identify Theileria and Anaplasma species in both hosts and vectors [10-17].

The Mongolian gazelle, an endemic ungulate species designated a threatened species by the World Conservation Union, is facing human and livestock disturbances of varying intensity in northern China. Although several studies have demonstrated that various Theileria, Babesia, Ehrlichia, and Anaplasma species circulate among sheep, goats, cattle, cervids, and humans in China, almost no data are available on the possible role of $P$. gutturosa as a host organism. The aim of this study was to detect and identify Theileria and Anaplasma spp. in P. gutturosa, a 
potential natural host of animal theileriosis and anaplasmosis in China.

\section{Methods}

\section{Sample collection}

The region investigated in China is located at latitudes 3503 ' 3555 ' north and longitudes 10537 ' 10808 ' east. The study was performed in April 2014. A total of 92 blood samples were collected randomly from P. gutturosa, and 242 ticks were collected from both $P$. gutturosa and grass in its environment. Of them, 30 unfed adult ticks were collected directly from grass in the gazelles environment; 212 engorged nymph ticks collected from P. gutturosa were kept at $28 \mathrm{C}$ and $80-90 \%$ relative humidity during molt, until nymph ticks were molted into adult ticks. All of adults were identified with Tengs methods [18]. Blood smears were prepared from the ear blood of every P. gutturosa individual. During the blood collection process, cases of suspected theileriosis or anaplasmosis were investigated. Theileriosis and/or anaplasmosis should be suspected in tick-infested animals with fever, enlarged lymph nodes (theileriosis only), anemia, and jaundice.

\section{Microscopic analysis of blood smears}

The blood smears were air-dried, fixed in methanol, stained with a $10 \%$ solution of Giemsa in phosphatebuffered saline ( $\mathrm{pH} 7.2)$, and then analyzed microscopically and photographed (Figure 1).

\section{DNA extraction}

Genomic DNA was extracted from the 92 whole blood samples and 222 tick samples using a genomic DNA extraction kit (Qiagen, Hilden, Germany), according to the manufacturers instructions. The DNA yields were determined with a NanoDrop 2000 spectrophotometer (Nanodrop Technologies, Wilmington, DE, USA).

\section{Molecular detection of Theileria and Anaplasma using species-specific primers}

PCR was used to detect and identify Theileria and Anaplasma spp. in P. gutturosa with the species-specific

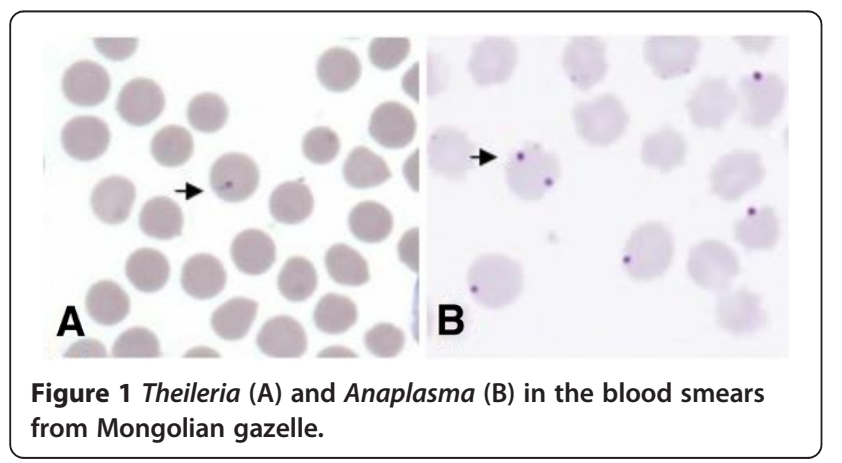

primers shown in Table $1[10,11,14-17]$. The PCR reactions were performed in an automatic DNA thermocycler (Bio-Rad, Hercules, CA, USA) and the PCR products were used to assess the presence of specific bands indicative of Theileria and Anaplasma.

The DNA fragments were sequenced by the GenScript Corporation (Piscataway, NJ, USA). Representative sequences of the $18 \mathrm{~S}$ rDNA/16S rDNA (or msp4) genes of the Theileria and Anaplasma spp. newly identified in this study were deposited in the GenBank database of the National Center for Biotechnology Information (NCBI) (http://www.ncbi.nlm.nih.gov/genbank/).

\section{Sequence alignments and phylogenetic analyses}

The MegAlign component of the Lasergene program version 4.01 (DNASTAR) was used to generate multiple sequence alignments with the ClustalW algorithm (www. clustal.org/) and for the phylogenetic analyses using the neighbor-joining method. A phylogenetic tree was constructed (Figure 2) based on the Theileria and Babesia 18S rDNA gene sequences determined in this study, and others obtained from the GenBank database under accession numbers: KM186951 KM186957, AY262118, JX469515, JF719832, AY661512, JF719834, EU274472, EU277003, AY260172, FJ603460, AY726011, KJ188212, EU083800, FJ426369, AY262120, KJ188228, Z15105, AY081192, AY260179, AY260176, GQ304524, AY260178, and HQ 264112. Another phylogenetic tree was constructed (Figure 3) based on sequences of the Anaplasma and Ehrlichia 16S RNA genes under the following accession numbers: KM186935 KM186937, KM186940, KM186944, KM 186947-KM186950, KM246795, KM246796, KM227012, HQ913644, HM131218, JX092094, JN558819, AY077619, EU439943, KM246802, AB196721, AY837736, KC484563, KJ639880, JQ917879, AF414869, NR_074356, KC479022, KC479024, and KJ659037.

\section{Ethical approval}

This study was approved by the Animal Ethics Committee of Lanzhou Veterinary Research Institute, CAAS (No. LVRIAEC2013-010). The use of these field samples was approved by the Animal Ethics Procedures and Guideline of China.

\section{Results}

\section{Tick identification}

In this study, all 242 ticks were collected from P. gutturosa or grass in its environment in north-western China. The identification result showed that the adult ticks were either Haemaphysalis longicornis $(\mathrm{n}=130$ : 86 female; 44 male) or Dermacentor niveus ( $\mathrm{n}=112$ : 78 female; 34 male). The whole DNA of $120 \mathrm{H}$. longicornis ticks and $102 \mathrm{D}$. niveus ticks was extracted. 
Table 1 Sequences of the oligonucleotide primers used in this study

\begin{tabular}{|c|c|c|c|c|c|}
\hline \multirow[t]{2}{*}{ Pathogen } & \multirow{2}{*}{$\begin{array}{l}\text { Target } \\
\text { gene }\end{array}$} & \multicolumn{2}{|l|}{ Primers } & \multirow{2}{*}{$\begin{array}{l}\text { Final amplicon } \\
\text { size (bp) }\end{array}$} & \multirow[t]{2}{*}{ References } \\
\hline & & Primer name & Oligonucleotide sequences (5-3) & & \\
\hline \multirow[t]{2}{*}{ Anaplasma \& Ehrlichia } & $16 \mathrm{~S}$ rRNA & EC9 & TACCTTGTTACGACTT & 1462 & Kawahara et al., 2006 [10] \\
\hline & & EC12A & TGATCCTGGCTCAGAACGAACG & & \\
\hline \multirow[t]{2}{*}{ A. bovis } & $16 \mathrm{~S}$ rRNA & AB1f & CTCGTAGCTTGCTATGAGAAC & 551 & Kawahara et al., 2006 [10] \\
\hline & & $A B 1 r$ & TCTCCCGGACTCCAGTCTG & & \\
\hline \multirow[t]{2}{*}{ A. phagocytophilum } & $16 \mathrm{~S}$ rRNA & SSAP2f & GCTGAATGTGGGGATAATTTAT & 641 & Kawahara et al., 2006 [10] \\
\hline & & SSAP2r & ATGGCTGCTTCCTTTCGGTTA & & \\
\hline \multirow[t]{2}{*}{ A. marginale } & msp4 & Amargmsp4 F & CTGAAGGGGGAGTAATGGG & 344 & Torina et al., 2012 [11] \\
\hline & & Amargmsp4 R & GGTAATAGCTGCCAGAGATTCC & & \\
\hline \multirow[t]{2}{*}{ A. ovis } & msp4 & Aovismsp4 F & TGAAGGGAGCGGGGTCATGGG & 347 & Torina et al., 2012 [11] \\
\hline & & Aovismsp4 R & GAGTAATTGCAGCCAGGGACTCT & & \\
\hline \multirow[t]{2}{*}{ Hemoparasite } & $18 \mathrm{~S}$ rRNA & Primer A & AACCTGGTTGATCCTGCCAGT & 1750 & Medlin et al., 1988 [14] \\
\hline & & Primer B & GATCCTTCTGCAGGTTCACCTAC & & \\
\hline \multirow[t]{2}{*}{ Theileria } & $18 \mathrm{~S}$ rRNA & 989 & AGTTTCTGACCTATCAG & 1100 & Allosop et al., 1993 [15] \\
\hline & & 990 & TTGCCTTAAACTTCCTTG & & \\
\hline \multirow[t]{2}{*}{ T. luwenshuni } & $18 \mathrm{~S}$ rRNA & $\mathrm{T} \mid 310$ & GGTAGGGTATTGGCCTACTGA & 340 & Yin et al., 2008 [16] \\
\hline & & T1680 & TCATCCGGATAATACAAGT & & \\
\hline \multirow[t]{2}{*}{ Babesia } & $18 \mathrm{~S}$ rRNA & Babesia F & TGTCTTGAATACTT(C/G)AGCATGGAA & 950 & Ramos et al., 2010 [17] \\
\hline & & Babesia R & CGACTTCTCCTITAAGTGATAAC & & \\
\hline
\end{tabular}

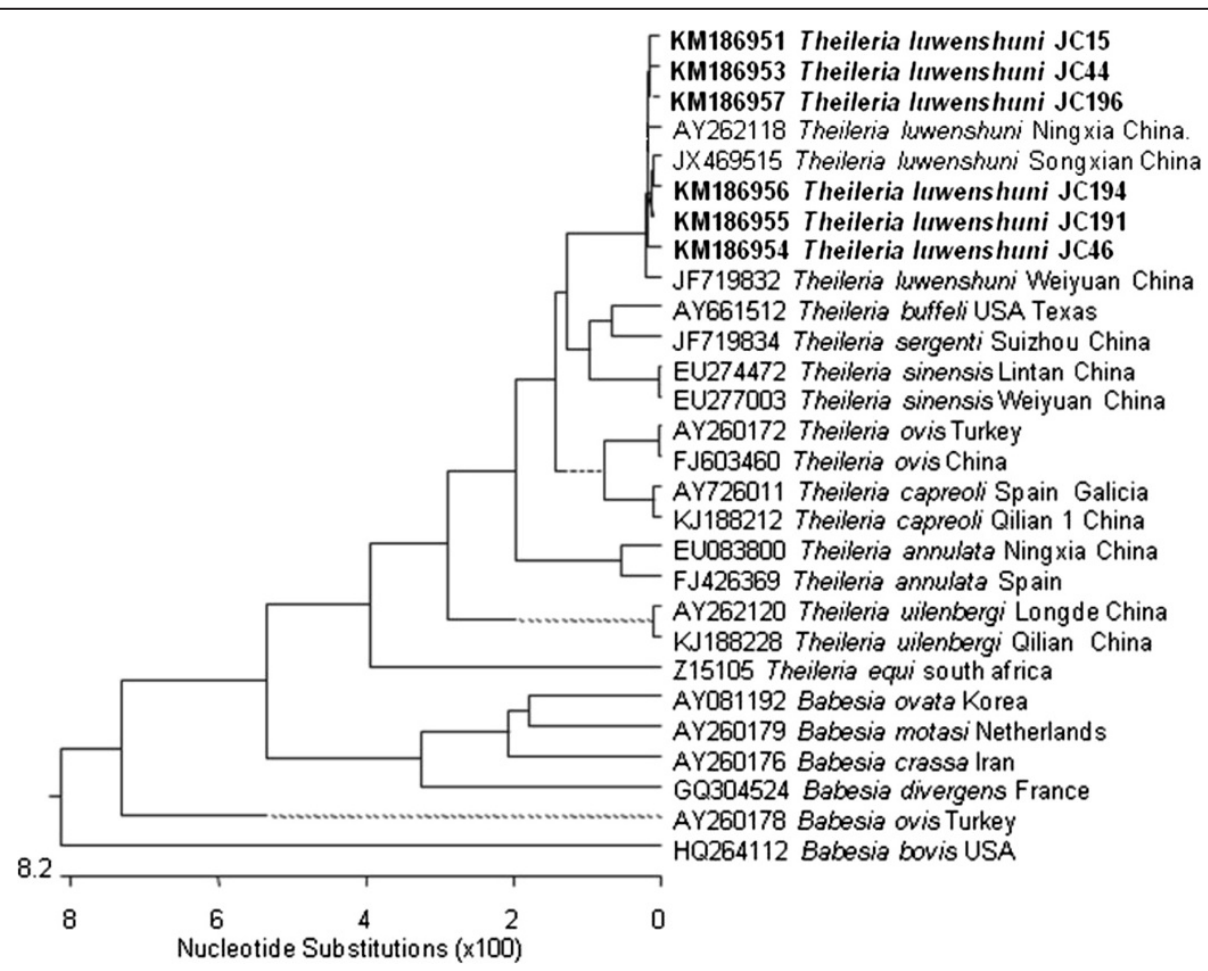

Figure 2 Phylogenetic tree of Theileria and Babesia based on 18S rDNA sequences. 


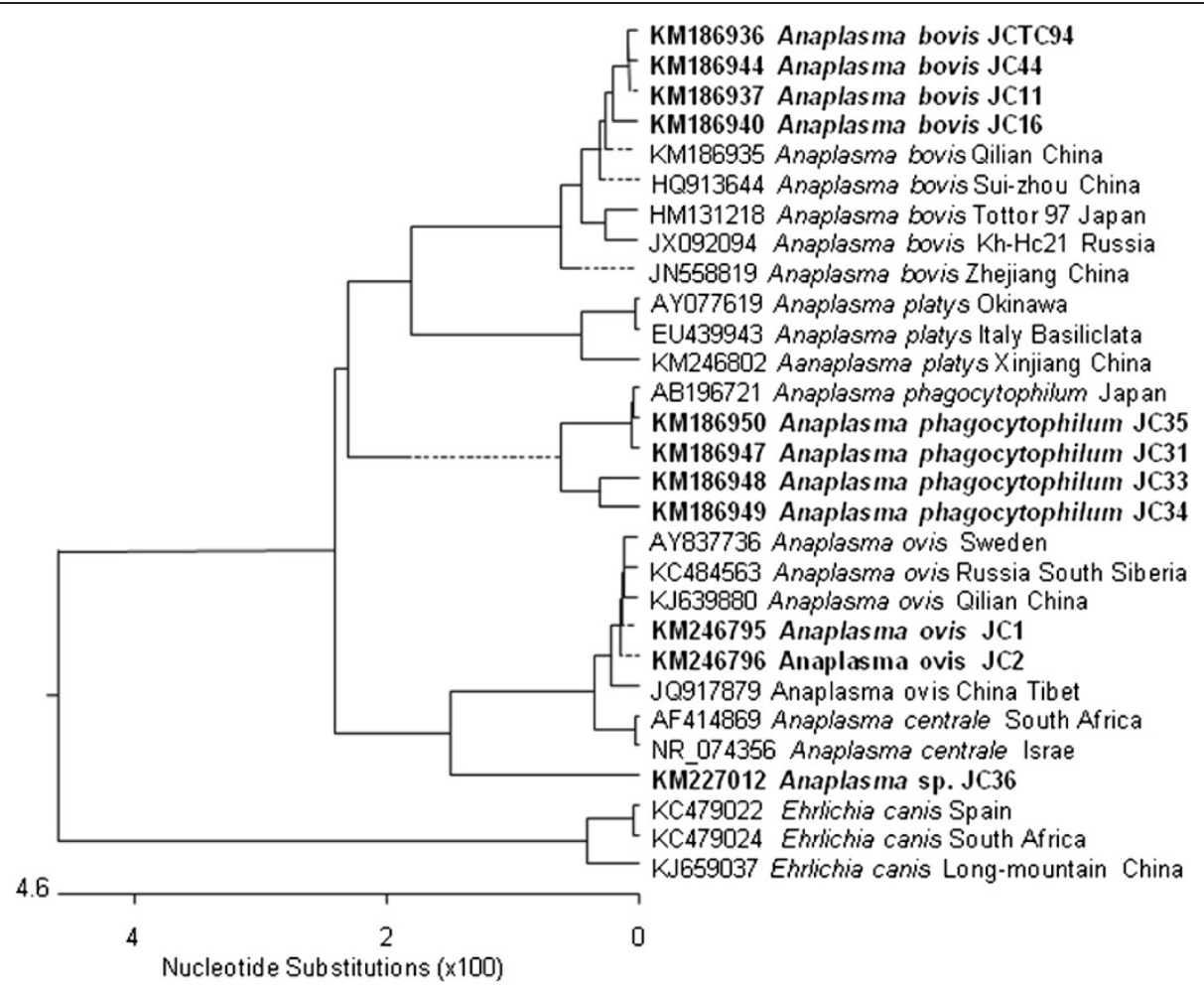

Figure 3 Phylogenetic tree of Theileria and Babesia based on 16S rDNA sequences.

Microscopic examination of blood smears

Theileriosis and anaplasmosis was present in $50 \%$ of the gazelles tested (46/92). Theileria and Anaplasma infections were observed microscopically in $87.0 \%$ (80/92), and $13.0 \%(12 / 92)$ of the blood smears from $P$. gutturosa individuals, respectively (Figure 1). All infected animals exhibited low levels of parasitemia, with $0.016 \%$ for Theileria and 0.01 4\% for Anaplasma.

PCR detection of Theileria and Anaplasma with speciesspecific primer sets

PCR analysis revealed that the prevalence of T. luwenshuni, A. bovis, A. phagocytophilum, and A. ovis in P. gutturosa was $97.8 \%, 78.3 \%, 65.2 \%$, and $52.2 \%$, respectively. Their prevalence in $H$. longicornis was $80.0 \%, 66.7 \%, 76.7 \%$, and $0 \%$, respectively, and their prevalence in $D$. niveus was $88.2 \%, 35.3 \%, 88.2 \%$, and $58.8 \%$, respectively (Table 2 ). No
Babesia sp. was found in P. gutturosa, H. longicornis, or D. niveus. Only one (4.3\%) of the 92 samples from P. gutturosa was positive for $R$. raoultii.

\section{Amplification of the $18 \mathrm{~S} / 16 \mathrm{~S}$ rDNA or msp4 genes and their accession numbers}

The nearly full-length $18 \mathrm{~S}$ rDNA sequences of $T$. luwenshuni were $1745 \mathrm{bp}$ with the primers $\mathrm{A} / \mathrm{B}$, and the accession numbers are KM186951 KM186957. The nearly fulllength 16S rDNA sequences were 1457 bp in $A$. bovis (KM186936 KM186944), 1458 bp in A. phagocytophilum (KM186947 KM186950), and 1456 bp in A. ovis (KM246795 and KM246796) with primers EC12/EC12A, which are specific for Anaplasma and Ehrlichia spp. An unknown Anaplasma sp. was isolated and its accession number was KM227012. The msp4 gene PCR products were 551 bp for A. bovis (KM226988, KM226999, KM227002,

Table 2 Prevalence of Theileria and Anaplasma in Procapra gutturosa and ticks in China

\begin{tabular}{|c|c|c|c|c|c|c|c|}
\hline \multirow[t]{3}{*}{ Host } & \multirow[t]{3}{*}{$\begin{array}{l}\text { No. of } \\
\text { samples }\end{array}$} & \multicolumn{6}{|c|}{$\begin{array}{l}\text { The prevalence of Theileria and Anaplasma in Procapra gutturosa and Ticks by PCR and Microscopic } \\
\text { Examination }\end{array}$} \\
\hline & & \multicolumn{2}{|c|}{ By Microscopic Examination (ME) } & \multicolumn{4}{|l|}{ By PCR } \\
\hline & & Theileria spp. & Anaplasma spp. & T. luwenshuni & A. bovis & A. phagocytophilum & A. ovis \\
\hline Procapra gutturosa & 92 & $87.0 \%(80 / 92)$ & $13.0 \%(12 / 92)$ & $97.8 \%(90 / 92)$ & $78.3 \%(72 / 92)$ & $65.2 \%(60 / 92)$ & $52.2 \%(48 / 92)$ \\
\hline H. Longicornis & 120 & / & / & $80.0 \%(96 / 120)$ & $66.7 \%(80 / 120)$ & $76.7 \%(92 / 120)$ & $0 \%$ \\
\hline Dermacentor niveus & 102 & / & / & $88.2 \%(90 / 102)$ & $35.3 \%(36 / 102)$ & $88.2 \%(90 / 102)$ & $58.8 \%(60 / 102)$ \\
\hline
\end{tabular}


and KM227003), 641 bp for A. phagocytophylum (KM227007 KM227009), and 347 bp for $A$. ovis (KM227005 and KM227006) when species-specific primers were used.

\section{Sequence alignments and phylogenetic analyses}

The phylogenetic tree based on the Theileria and Babesia $18 \mathrm{~S}$ rDNA sequences showed that only one pathogen was detected, which was placed in the T. luwenshuni cluster (Figure 2). The phylogenetic tree based on the $16 \mathrm{~S}$ rDNA sequences of Anaplasma and Ehrlichia revealed four pathogens existed and they were A. bovis, A. phagocytophilum, A. ovis, and Anaplasma sp., respectively, in the blood samples from P. gutturosa roaming northern China (Figure 3).

\section{Discussion}

To the best of our knowledge, this study is the first to report the prevalence of theileriosis and anaplasmosis in $P$. gutturosa in China. Molecular screening of P. gutturosa in northern China showed that the most prevalent Theileria and Anaplasma species were, in descending order: $T$. luwenshuni $>A$. bovis $>A$. phagocytophilum $>A$. ovis. No other Theileria sp. or Anaplasma sp. was detected in $P$. gutturosa. The prevalence of T. luwenshuni and A. bovis in $P$. gutturosa was higher than their prevalence in $H$. longicornis or $D$. niveus. However, the prevalence of $A$. phagocytophilum was, in descending order: D. niveus $>H$. longicornis $>P$. gutturosa. We speculate that persistent pathogen reservoirs with high infection rates are well established in P. gutturosa in northern China.

Anaplasma bovis infections of cattle have been reported predominantly in African countries, and there have been few reports of bovine $A$. bovis infections in China. Recently, A. ovis and $A$. bovis were reported in goats in central and southern China, and $A$. marginale was detected in cattle in southern China [9]. A. bovis and A. ovis have also been reported in red deer, sika deer, and $D$. everestianus in northwestern China [12]. In Japan, $A$. bovis and $A$. centrale have been detected in wild deer and $H$. longicornis ticks on Honshu Island, Japan [10]; A. bovis and A. phagocytophilum were initially detected in cattle on Yonaguni Island, Okinawa, Japan [19]. Therefore, $H$. megaspinosa is considered a dominant vector tick species for both these species in cattle in Japan [20]. In this study, four Anaplasma spp. (A. bovis, A. ovis, A. phagocytophilum, and an Anaplasma sp.) were detected in P. gutturosa. Rickettsia raoultii was also detected for the first time in P. gutturosa in China.

In this study, all 242 ticks were collected from gazelle or from their environment in the investigated area. They consisted of $H$. longicornis and D. vineus. Theileria luwenshuni and Anaplasma spp. (including A. bovis, A. phagocytophilum, A. ovis, and Anaplasma sp.) were detected and identified by PCR. Therefore, we speculate that these ticks play an important role as natural vectors of Anaplasma spp. in northern China. Theileria luwenshuni were first reported in sheep and goats, and widely distributed in north-western China [21]; recently, it was also reported in sheep and goats in central and southern China [22-24]. T. luwenshuni can be transmitted by $H$. qinghaiensis and $H$. longicornis in north-western China [25], but only $H$. longicornis and $D$. niveus were found in this study. Therefore, $H$. longicornis must play an important role as a natural vector of $T$. luwenshuni in P. gutturosa in northern China. However, whether T. luwenshuni can be transmitted by $D$. niveus remains to be determined.

\section{Conclusion}

Our results provide important data that extend our understanding of the epidemiology of theileriosis and anaplasmosis, and should facilitate the implementation of measures to control the transmission of Theileria and Anaplasma among P. gutturosa and other relative ruminants in China. Clarification of the role of P. gutturosa as a reservoir host for some Theileria and Anaplasma species is critical in determining whether P. gutturosa contributes to the spread of ruminant theileriosis and anaplasmosis in China.

\section{Competing interests \\ The authors declare that they have no competing interests.}

\section{Authors contributions}

QR and GG collected the samples; $Y L, Z C, Z L$, and $J$ performed the molecular genetic studies; JY, QL, YL, and SC performed the sequence alignments; YL, JL, and $\mathrm{HY}$ drafted the manuscript. All authors have read and approved the final manuscript.

\section{Acknowledgments}

This study was supported financially by the Natural Sciences of Foundation China (no. 31272556, no. 31372432, no. 31101621, no. 31201899), ASTIP, FRIP (2014ZL010), CAAS, Creative Research Groups of Gansu Province (no. 1210RJIA006), NBCIS (CARS-38), 948 (2014-S05), the Special Fund for Agro-scientific Research in Public Research (no. 201303035, no. 201303037), MOA; 973 Program (2010CB530206), Basic Research Program (CRP no. 16198/ R0), Supporting Program (2013BAD12B03, 2013BAD12B05), Specific Fund for Sino-Europe Cooperation, MOST, China; and the State Key Laboratory of Veterinary Etiological Biology Project. The research was also supported by CRP (no. 16198/RO IAEA) and PIROVAC (KBBE-3-245145) of the European Commission. Thanks for the revision by Edanz Group Ltd (China).

Received: 24 September 2014 Accepted: 18 December 2014 Published online: 21 December 2014

\section{References}

1. Dumler JS, Choi KS, Garcia-Garcia JC, Barat NS, Scorpio DG, Garyu JW, Grab DJ, Bakken JS: Human granulocytic anaplasmosis and Anaplasma phagocytophilum. Emerg Infect Dis 2005, 11:1828 1834.

2. Chen SM, Dumler JS, Bakken JS, Walker DH: Identification of a granulocy to tropic Ehrlichia species as the etiologic agent of human disease. J Clin Microbiol 1994, 32:589 595.

3. Aktas M, Altay K, Dumanli N: Molecular detection and identification of Anaplasma and Ehrlichia species in cattle from Turkey. Ticks Tick Borne Dis 2011, 2:62 65.

4. Aktas M, Altay K, Ozubek S, Dumanli N: A survey of ixodid ticks feeding on cattle and prevalence of tick-borne pathogens in the Black Sea region of Turkey. Vet Parasitol 2012, 187:567 571.

5. Aktas M: A survey of ixodid tick species and molecular identification of tick-borne pathogens. Vet Parasitol 2014, 200:276 283. 
6. Silaghi C, Woll D, Hamel D, Pfister K, Mahling M, Pfeffer M: Babesia spp. and Anaplasma phagocytophilum in questing ticks, ticks parasitizing rodents and the parasitized rodents analyzing the host-pathogen-vector interface in a metropolitan area. Parasit Vectors 2012, 5:191.

7. Silveira JA, Rabelo EM, Ribeiro MF: Molecular detection of tick-borne pathogens of the family Anaplasmataceae in Brazilian brown brocket deer (Mazama gouazoubira, Fischer, 1814) and marsh deer (Blastocerus dichotomus, Illiger, 1815). Transbound Emerg Dis 2012, 59:353 360.

8. Sarih M, M Ghirbi Y, Bouattour A, Gern L, Baranton G, Postic D: Detection and identification of Ehrlichia spp. in ticks collected in Tunisia and Morocco. J Clin Microbiol 2005, 43:1127 1132.

9. Liu Z, Ma M, Wang Z, Wang J, Peng Y, Li Y, Guan G, Luo J, Yin H: Molecular survey and genetic identification of Anaplasma species in goats from central and southern China. Appl Environ Microbiol 2012, 78:464 470.

10. Kawahara M, Rikihisa Y, Lin Q, Isogai E, Tahara K, Itagaki A, Hiramitsu Y, Tajima T: Novel genetic variants of Anaplasma phagocytophilum, Anaplasma bovis, Anaplasma centrale, and a novel Ehrlichia sp. in wild deer and ticks on two major islands in Japan. Appl Environ Microbiol 2006, 72:1102 1109.

11. Torina A, Agnone A, Blanda V, Alongi A, D'Agostino R, Caracappa S, Marino AM, Di Marco V, de la Fuente J: Development and validation of two PCR tests for the detection of and differentiation between Anaplasma ovis and Anaplasma marginale. Ticks Tick Borne Dis 2012, 3:283 287.

12. Li Y, Chen Z, Liu Z, Liu J, Yang J, Li Q, Li Y, Cen S, Guan G, Ren Q, Luo J, Yin H: Molecular identification of Theileria parasites of north-western Chinese Cervidae. Parasit Vectors 2014, 14(7):225.

13. Chen Z, Liu Q, Liu JQ, Xu BL, Lv S, Xia S, Zhou XN: Tick-borne pathogens and associated co-infections in ticks collected from domestic animals in central China. Parasit Vectors 2014, 7:237.

14. Medlin L, Elwood HJ, Stickel S, Sogin ML: The characterization of enzymatically amplified eukaryotic 16S-like rRNA-coding regions. Gene 1988, 71:491 499.

15. Allsopp BA, Baylis HA, Allsopp MT, Cavalier-Smith T, Bishop RP, Carrington DM, Sohanpal B, Spooner P: Discrimination between six species of Theileria using oligonucleotide probes which detect small subunit ribosomal RNA sequences. Parasitol 1993, 107:157 165.

16. Yin H, Liu Z, Guan G, Liu A, Ma M, Ren Q, Luo J: Detection and differentiation of Theileria luwenshuni and T. uilenbergi infection in small ruminants by PCR. Transbound Emerg Dis 2008, 55:233 237.

17. Ramos CM, Cooper SM, Holman PJ: Molecular and serologic evidence for Babesia bovis-like parasites in white-tailed deer (Odocoileus virginianus) in south Texas. Vet Parasitol 2010, 172:214 220.

18. Teng KF, and Jiang ZI: Economic Insect Fauna of China. Fasc 39, Acari: Ixodidae. Science Press 1991, 158181.

19. Ooshiro M, Zakimi S, Matsukawa Y, Katagiri Y, Inokuma H: Detection of Anaplasma bovis and Anaplasma phagocytophilum from cattle on Yonaguni Island, Okinawa, Japan. Vet Parasitol 2008, 154:360 364.

20. Yoshimoto K, Matsuyama Y, Matsuda H, Sakamoto L, Matsumoto K, Yokoyama N, Inokuma H: Detection of Anaplasma bovis and Anaplasma phagocytophilum DNA from Haemaphysalis megaspinosa in Hokkaido, Japan. Vet Parasitol 2010, 168:170 172.

21. Yin H, Schnittger L, Luo JX, Ulrike S, Ahmed J: Ovine theileriosis in China: a new look at an old story. Parasitol Res 2007, 101:S191 S195.

22. Li Y, Zhang X, Liu Z, Chen Z, Yang J, He H, Guan G, Liu A, Ren Q, Niu Q, Liu J, Luo J, Yin H: An epidemiological survey of Theileria infections in small ruminants in central China. Vet Parasitol 2014, 200:198 202.

23. Yang Y, Mao Y, Kelly P, Yang Z, Luan L, Zhang J, Li J, El-Mahallawy HS, Wang C: A: pan-Theileria FRET-qPCR survey for Theileria spp. in ruminants from nine provinces of China. Parasit Vectors 2014, 7(1):413.

24. Wu XB, Na RH, Wei SS, Zhu JS, Peng HJ: Distribution of tick-borne diseases in China. Parasit Vectors 2013, 6:119.

25. Li Y, Luo J, Liu Z, Guan G, Gao J, Ma M, Dang Z, Liu A, Ren Q, Lu B, Liu J, Zhao H, Li J, Liu G, Bai Q, Yin H: Experimental transmission of Theileria sp. (China 1) infective for small ruminants by Haemaphysalis longicornis and Haemaphysalis qinghaiensis. Parasitol Res 2007, 101:533 538.

\section{Submit your next manuscript to BioMed Central and take full advantage of:}

$\otimes$ Convenient online submission

$凶$ Thorough peer review

$\otimes$ No space constraints or color $₫$ gure charges

$\triangle$ Immediate publication on acceptance

QInclusion in PubMed, CAS, Scopus and Google Scholar

$\triangle$ Research which is freely available for redistribution

Submit your manuscript at www.biomedcentral.com/submit
( BioMed Central 